\title{
2 Exploring the relationship between EQ-5D, DLQI and PASI, 3 and mapping EQ-5D utilities: a cross-sectional study in psoriasis 4 from Hungary
}

\author{
Emese Herédi • Fanni Rencz • Orsolya Balogh • László Gulácsi • Krisztina Herszényi • \\ Péter Holló · Hajnalka Jókai · Sarolta Kárpáti · Márta Péntek · Éva Remenyik · \\ Andrea Szegedi $\cdot$ Valentin Brodszky
}

Received: 12 February 2014/ Accepted: 31 March 2014

(C) Springer-Verlag Berlin Heidelberg 2014

\begin{abstract}
Background There is a growing interest in policy making for using utility measures and identifying algorithms to convert disease-specific measures into utilities.

Objectives To analyse the relationship between EQ-5D, Dermatology Life Quality Index (DLQI) and Psoriasis Area and Severity Index (PASI) in psoriasis. To transform DLQI scores, and key clinical, demographic and health service utilisation variables into utilities.

Methods A cross-sectional questionnaire survey of 200 consecutive adult patients with moderate to severe psoriasis was carried out in two Hungarian university clinics. The relationship between the outcome measures were analysed with correlations and with the known-groups method. Bivariate and multivariate regression algorithms on EQ-5D scores were formulated.
\end{abstract}

Emese Herédi and Fanni Rencz have contributed equally to this work.

E. Herédi · É. Remenyik · A. Szegedi

Departments of Dermatology and Dermatological Allergology,

University of Debrecen, Nagyerdei krt. 98., Debrecen 4032,

Hungary

F. Rencz · O. Balogh · L. Gulácsi · M. Péntek ·

V. Brodszky ( $\square)$

Department of Health Economics, Corvinus University of

Budapest, Fővám tér 8., Budapest 1093, Hungary

e-mail: valentin.brodszky@uni-corvinus.hu

\section{F. Rencz}

Doctoral School of Clinical Medicine, Semmelweis University,

Üllői út 26., Budapest 1085, Hungary

\section{K. Herszényi · P. Holló · H. Jókai · S. Kárpáti}

Department of Dermatology, Venereology and

Dermatooncology, Semmelweis University, Mária u. 41., Budapest 1085, Hungary
Results The mean age of respondents was 51 years $(\mathrm{SD}=12.9), 68.5 \%$ were male, and $51.5 \%$ received biological therapy. Median EQ-5D, DLQI, and PASI scores were $0.73,3.0$, and 3.45 , respectively. EQ-5D showed a moderate correlation with the DLQI and with the PASI $\left(r_{\mathrm{s}}=-0.48\right.$ and $\left.-0.43, p<0.05\right)$. Strong correlation was found between DLQI and PASI $\left(r_{\mathrm{s}}=0.81, p<0.05\right)$. DLQI and PASI discriminated better among groups categorised by the localisation of the lesions than EQ-5D. Presence of psoriasis on the neck and/or décolletage was associated with the greatest health related quality of life (HRQOL) impairment. Ten variables were incorporated in a multivariate algorithm that accounted for $48.8 \%$ of EQ5D variance (ANOVA $p<0.001$ ).

Conclusions This study provided the first evidence that patients with visible psoriatic lesions have significantly worse HRQOL compared to those with non-visible lesions, measured not only with DLQI but also with EQ-5D. In addition to demographic and clinical variables, our model included health service utilisation variables related to psoriasis, and explained higher proportion of EQ-5D variance than any previous findings in the literature.

Keywords Psoriasis · Quality of life · EQ-5D · DLQI · Mapping

JEL Classification I19

\section{Introduction}

Psoriasis is a chronic immune-mediated inflammatory disease of the skin with various presentations and clinical courses. It is estimated to affect approximately 0.73-2.9\% of the population throughout Europe [1]. Extra-cutaneous

\begin{tabular}{|l|lll|}
\hline Journal : Large $\mathbf{1 0 1 9 8}$ & Dispatch : 2-5-2014 & Pages : 9 \\
Article No. : $\mathbf{6 0 0}$ & $\square$ LE & $\square$ TYPESET \\
MS Code : Supplement14_Paper12 & $\sim_{\mathrm{CP}}$ & $\checkmark$ DISK \\
\hline
\end{tabular}


manifestations such as arthritis, cardiovascular diseases or mental disorders are often associated with psoriasis [1]. To date, there is no definitive cure for the disease, and, therefore, patients usually need long-term treatment. Severe psoriasis has a profound impact on patients' health related quality of life (HRQOL) encompassing physical, psychological, and socio-economic levels [2].

Economic evaluations require data on HRQOL on preference-based measures that capture preference weights (called a utility, in terms of desirability) about values of different health states. Also, in many countries utility measures are required for reimbursement decisions. EQ-5D is the most commonly used utility measure in health economic analyses, however, it is rarely administered in clinical trials. Therefore, there is a demand for crosswalking (or mapping) algorithms to estimate EQ-5D utility scores from other HRQOL measures.

In recent years, introduction of biological agents (adalimumab, etanercept, infliximab, and ustekinumab) opened up new horizons in the treatment of patients with severe psoriasis. Compared to standard treatment, they proved clinical efficacy, but their use is associated with much higher costs and societal burden as well $[3,4]$. Due to biologicals, HRQOL measures should be able to face a new patient population with better health state, with currently unexplored possible predictors of HRQOL and with new expectations of treatment outcomes.

There have been continuous discussions concerning the most appropriate, valid, sensitive, and reliable HRQOL assessment tool in psoriasis [5]. Dermatology Life Quality Index (DLQI), Psoriasis Area and Severity Index (PASI), and Short Form-36 (SF-36) are the most widely used instruments in psoriasis. Although these are focusing on different aspects of HRQOL, several overlaps exist between them.

DLQI was the first disease-specific questionnaire in dermatology with 20 years of use in clinical trials and in everyday clinical practice by now. It has been considered a simple, valid, and reliable outcome measure is psoriasis [6]. Nevertheless, from the perspective of health economics, a major disadvantage of DLQI has to be addressed. Due to it is not a preference-based measure, it does not enable one to calculate utilities for economic evaluations.

Over the past decade, the literature on mapping the general measure EQ-5D in different diseases has rapidly grown. According to the University of Oxford HERC online database of mapping studies [7], only two papers and a conference abstract have been published about mapping EQ-5D in psoriasis, so far [8-10]. All these researches investigated the relationship between the dermatology-specific DLQI questionnaire and the EQ-5D index.
Recent evidences suggest a significant moderate correlation between EQ-5D and DLQI global scores [8, 11]. Prior mapping studies could explain only $27-31.3 \%$ of the variance of EQ-5D [8-10]. Consequently, almost $70 \%$ of the possible predictors of EQ-5D in psoriasis has still remained hidden.

The objectives of this present cross-sectional study are, at first, to analyse correlations between the widely used HRQOL and disease severity instruments of psoriasis and compare their capacity to distinguish among patients' severity groups; secondly, to seek for new possible predictors of HRQOL to establish mapping models on EQ-5D score and on the visual analogue scale (EQ VAS).

\section{Methods}

Patients

Between September 2012 and May 2013, a cross-sectional questionnaire survey of consecutive adult psoriasis patients from two Hungarian university clinics was carried out. The number of participants was limited to approximately 100 patients from each clinic. Patients included were required to be 18 years or older and to have been diagnosed with moderate to severe psoriasis (PASI $>10$ or DLQI $>10$ or patient using systemic or biological treatment) 12 months or more before the inclusion to the study. Data were collected by dermatologists at Semmelweis University, Department of Dermatology, Venereology and Dermatooncology (Budapest) and at the University of Debrecen, Clinic of Dermatology. All patients were invited to participate by their physicians during outpatient visits and signed an informed consent form. The study was approved by the national research ethic committee (ETT-TUKEB 35183/2012-EKU).

Outcome measures and assessment

All participants and their physicians were asked to complete a self-designed questionnaire. Patients' questionnaires concerned demographic data, general health state, quality of life (EQ-5D, EQ VAS, DLQI, self-assessed disease severity VAS) affected body sites, and disease duration. Dermatologists' questionnaires were based on the patients' clinical type of psoriasis, PASI, psoriasis treatments in the last 12 months, current clinical outcomes, and physician's' global assessment of disease activity visual analogue scale (PGA VAS).

Quality of life was captured by the validated Hungarian versions of EQ-5D questionnaire, by PGA VAS and by

\begin{tabular}{|l|lll|}
\hline & Journal : Large 10198 & Dispatch : 2-5-2014 & Pages : 9 \\
Article No. : 600 & $\square$ LE & $\square$ \\
MS Code : Supplement14_Paper12 & $\checkmark_{\mathrm{CP}}^{\text {TYPESET }}$ \\
\hline
\end{tabular}


disease-specific DLQI. Clinical severity of psoriasis was assessed by using Psoriasis Area and Severity Index (PASI-72) and patients' self-assessed disease severity VAS. Questions included if there were any GP visit(s) in the last months, dermatologist visit(s) in the last three months and hospitalisation(s) in the last 12 months. Necessity of home help (professional or informal, e.g., family members) in the last one month and work impairment due to psoriasis were also recorded.

EQ-5D consists of a five-item instrument to assess general HRQOL (mobility, self-care, usual activities, pain/ discomfort, and anxiety/depression), and of a visual analogue scale (EQ VAS). In the current survey, EQ-5D-3L version was used in which each dimension has three response levels (no problems, some problems, and severe problems), accordingly, $3^{5}=243$ combinations of health states are possible. Due to lack of evaluated Hungarian tariffs, the UK weights were applied to calculate global EQ-5D scores; thus, utility outcomes can range from -0.59 to +1 , where -0.59 is corresponding to the worst and +1 corresponding to the best possible quality of life [12]. EQ VAS is a $20 \mathrm{~cm}$ long, vertical visual analogue scale with endpoints of ' 0 ' (worst possible health state) and ' 100 ' (best possible health state) recording patients' self-rating of their overall health, which as well enables determining utilities.

DLQI is a disease-specific self-assessment questionnaire validated for measuring HRQOL in psoriasis [13, 14]. The ten-item questionnaire's scale ranged from ' 0 ' to ' 30 ', where higher scores indicate greater disability experienced by patients. Each questions of DLQI scores quality of life impairment due to the dermatologic condition in a 4-point Likert scale, including aspects such as symptoms, side effects of treatment, daily activities, work or school, personal relationships, leisure activities, and feelings of embarrassment.

PASI-72 (hereinafter PASI) is a quantitative rating scale for psoriasis based on the severity of the lesions and the size of psoriatic areas assessed by physicians. It is widely used both in clinical trials to measure clinical effectiveness and in routine care to evaluate treatment success. To calculate PASI scores, the body is divided into four sections based on the estimated area of the skin affected (head $=0.1$, upper extremities $=0.2$, trunk $=0.3$ and lower extremities $=0.4$ ). Each area is graded by itself from 0 to 6 , depending on the estimated percentage of the psoriatic involvement $(0=0 \%, 1 \leq 10 \%, 2=10-29 \%$, $3=30-49 \%, \quad 4=50-69 \%, \quad 5=70-89 \%, \quad$ and $6=90-100 \%$ ). Within each area, severity is judged by the presence of three clinical signs: erythema, induration, and desquamation (measured on a scale of $0-4$ ). Total PASI values range from 0 to 72 , with higher scores indicating greater disease severity.
Statistical analysis

Spearman's rank correlation was used to test associations between outcome measures.

Mann-Whitney $U$ test was performed to compare the differences in the distribution of EQ-5D, DLQI, and PASI.

The known-groups method was applied to compare the outcome measures ability to detect differences between groups with known attributes. Overall 11 categories, including clinical types, localisation and several medical records were selected for grouping variables. In each category we expected that patients responded 'Yes' to a question had worse scores in quality of life or in disease severity measures than those who responded 'No' (i.e., control group). To compare the means of the two groups, effect size (Cohen's $d$ ) was calculated by dividing the difference of the means by pooled standard deviation. The Cohen's $d$ is considered small if $0.2-0.5$, medium if $0.5-0.8$, or large if $>0.8$, respectively, where the measure with a higher value can better distinguish between groups [15].

To determine possible predictors of quality of life in psoriasis, age, disease duration, body mass index (BMI), and instruments that significantly correlated with EQ-5D, were enrolled as continuous variables. Additionally, those categorical variables were selected as possible predictors which proved a significant EQ-5D difference between their two possible outcomes (e.g., presence or absence of a clinical type, symptom or treatment). From this point forward, negative EQ-5D values were truncated to 0 . In a bivariate mapping model on EQ-5D score and on EQ VAS, only DLQI was included as an independent predictor of the target variables. Then, to find an optimal algorithm in a multivariate approach that can explain the highest proportion of variance, we included all the possible predictors, which were found to be in a significant relationship with the target variable.

Data were analysed using SPSS version 20.0 (SPSS Inc., Chicago, IL, USA). All the applied statistics were twosided with a significance level of $p<0.05$.

\section{Results}

Patient characteristics

Altogether 200 patients participated in the survey. Patient characteristics are described in Table 1.

The mean age was 51 years with male predominance $(68.5 \%)$. The mean disease duration was 22 years. Overall $159(79.4 \%)$ of the participants were overweight (BMI $\geq 25$ ). The most frequent type of psoriasis was chronic plaque psoriasis with 126 (63\%), followed by nail 
Table 1 Patient characteristics

\begin{tabular}{lrrlll}
\hline & $n$ & Mean & SD & Median & Range \\
\hline Age (years) & 200 & 51.24 & 12.9 & \multicolumn{1}{l}{53} & $21-85$ \\
$\begin{array}{l}\text { Psoriasis duration } \\
\quad \text { years) }\end{array}$ & 200 & 21.96 & 11.67 & 20.5 & $1-63$ \\
BMI (kg/m²) & 199 & 29.89 & 5.44 & 29.41 & $16.45-46.81$ \\
EQ-5D score (-0.594 & 192 & 0.69 & 0.31 & 0.73 & -0.43 to 1 \\
$\quad$ to 1) & & & & & \\
EQ VAS (0-100) & 196 & 64.43 & 21.34 & 70.00 & $0-100$ \\
DLQI (0-30) & 194 & 6.29 & 7.29 & 3.00 & $0-28$ \\
$\begin{array}{l}\text { PASI (0-72) } \\
\text { Physician's global }\end{array}$ & 200 & 8.01 & 10.01 & 3.45 & $0-49.5$ \\
$\quad 189$ & 23.39 & 28.24 & 7.00 & $0-100$ \\
$\quad \begin{array}{l}\text { assessment VAS } \\
\quad(0-100 \text { mm) }\end{array}$ & & & & & \\
$\begin{array}{l}\text { Self-assessed disease } \\
\quad \text { severity VAS }\end{array}$ & 199 & 34.84 & 33.33 & 20.00 & $0-100$ \\
$\quad(0-100$ mm) & & & & & \\
\hline
\end{tabular}

psoriasis $71(35.5 \%)$, scalp psoriasis 69 (34.5\%), psoriatic arthritis $57(28.5 \%)$, inverse psoriasis 18 (9\%), palmoplantar psoriasis $12(6 \%)$, erythrodermic psoriasis four $(2 \%)$, and guttate psoriasis two (2\%) (combinations are possible). In total, $50(25 \%)$ of the patients reported psoriasis involvement of the face, 36 (18\%) of the neck and/or décolletage, $83(41.5 \%)$ of the hands and/or palms, 69 $(34.5 \%)$ of the hand nails, $110(55 \%)$ of the forearms, and $134(67 \%)$ of the lower extremity, respectively. At the time of the survey, $59(29.5 \%)$ of the patients had no symptoms at all (i.e., PASI $=0$ ).

Among the included patients, $103(51.5 \%)$ received biological drug in monotherapy or combination therapy, 61 $(30.5 \%)$ systemic non-biological therapy, and 30 (15\%) only topical treatment at the time of the survey.

The distribution of scores in the applied quality of life instruments were skewed, thus, the median is considered a better measure for the centre. The medians of quality of life tools were 0.73 for EQ-5D, 70 for EQ VAS, 3 for DLQI global score and 3.45 for PASI, respectively.

Frequencies of health service utilisation variables, including medical examinations, types of treatment, and additional non-reimbursed services are described elsewhere [16].

\section{Comparison}

Results obtained from correlation analysis of the instruments are demonstrated in Table 2. EQ-5D score showed a moderate negative correlation with DLQI, PASI, PGA, and with patients self-assessed disease severity VAS $(0.29<$ Spearman's-rho $<0.5)$. A strong significant correlations were found among DLQI, PASI, PGA, and selfassessed disease severity VAS.
Table 2 Spearman's correlations between the outcome measures

\begin{tabular}{lllll}
\hline & $\begin{array}{l}\text { EQ-5D score } \\
(-0.59 \text { to } 1)\end{array}$ & DLQI & PASI & $\begin{array}{l}\text { PGA } \\
\text { VAS }\end{array}$ \\
\hline EQ VAS (0-100) & $0.56^{*}$ & $-0.43^{*}$ & $-0.42^{*}$ & $-0.42^{*}$ \\
DLQI (0-30) & $-0.48^{*}$ & - & $0.81^{*}$ & $0.80^{*}$ \\
PASI (0-72) & $-0.43^{*}$ & $0.81^{*}$ & - & $0.92^{*}$ \\
PGA VAS (0-100 mm) & $-0.42^{*}$ & $0.80^{*}$ & $0.92^{*}$ & - \\
Self-assessed disease & $-0.41^{*}$ & $0.78^{*}$ & $0.78^{*}$ & $0.79^{*}$ \\
$\quad$ severity VAS & & & & \\
$(0-100$ mm) & & & & \\
\hline
\end{tabular}

* Significant $p<0.05$. For DLQI and PASI ' 0 ' and for all other measures, the highest value is the best possible outcome

The differences between known-groups are presented in Table 3. As expected, in each category patients with more severe disease (responded 'Yes') reported significantly worse quality of life than the control group (Mann-Whitney $U$ test, $p<0.05)$. EQ-5D revealed the highest effect sizes in 4 out of the 11 examined categories: GP visit(s) in the last month, necessity of home help in the last month, and in the clinical types of palmoplantar psoriasis and psoriatic arthritis. Nevertheless, it was the least effective tool in capturing the variables of hospitalisation(s) in the last year, biological therapy and the localisations of psoriatic lesions. DLQI and PASI were able to discriminate between these groups better. Patients with visible lesions (on body areas uncovered by clothes-face, neck, décolletage, hands, palms, hand nails) reported poorer HRQOL than those without visible lesions measured with any instrument.

Mapping EQ-5D

A simple linear regression of DLQI onto both EQ-5D score and EQ VAS was performed: EQ-5D $=0.8-0.02 * \mathrm{DLQI}$ (adjusted $r^{2}=0.169$, ANOVA $p<0.001$ ), EQ VAS $=$ $71.23-1.07 *$ DLQI (adjusted $r^{2}=0.129, \quad$ ANOVA $p<0.001)$. Thus, DLQI global score explained $16.9 \%$ of the variance of EQ-5D and $12.9 \%$ of the variance of EQ VAS.

In order to establish a multivariate function, only those variables were applied which were previously tested and showed significant correlation (continuous variables) or significant EQ-5D difference among their outcomes (categorical variables) with the target indices. Thus, overall, 23 possible predictors of EQ-5D and 21 of EQ VAS were identified.

In the final stepwise multiple regression, ten out of the 23 possible predictors of EQ-5D and six out of the 21 possible predictors of EQ VAS were enrolled (see Table 4). The models are explaining $48.8 \%$ of EQ-5D

\begin{tabular}{|l|lll|}
\hline & Journal : Large 10198 & Dispatch : 2-5-2014 & Pages : 9 \\
Article No. : $\mathbf{6 0 0}$ & $\square$ LE & $\square$ TYPESET \\
MS Code : Supplement14_Paper12 & $\boldsymbol{\sim}_{\mathrm{CP}}$ & $\checkmark_{\text {DISK }}$ \\
\hline
\end{tabular}


Table 3 Differences in effect size (Cohen's $d$ ) between outcome measures with the known-groups method

\begin{tabular}{|c|c|c|c|c|c|c|c|c|c|c|c|c|}
\hline & \multicolumn{3}{|c|}{ EQ-5D** } & \multicolumn{3}{|c|}{ EQ-5D VAS } & \multicolumn{3}{|c|}{ DLQI } & \multicolumn{3}{|c|}{ PASI } \\
\hline & $n$ & Mean & $\begin{array}{l}\text { Effect } \\
\text { size }\end{array}$ & $n$ & Mean & $\begin{array}{l}\text { Effect } \\
\text { size }\end{array}$ & $n$ & Mean & $\begin{array}{l}\text { Effect } \\
\text { size }\end{array}$ & $n$ & Mean & $\begin{array}{l}\text { Effect } \\
\text { size }\end{array}$ \\
\hline \multicolumn{13}{|c|}{ Clinical type of psoriasis } \\
\hline \multicolumn{13}{|c|}{ Palmoplantar psoriasis } \\
\hline No & 152 & $0.71(0.29)$ & 1.2 & 162 & $63.59(21.08)$ & 0.63 & 160 & $6.41(7.37)$ & 0.69 & 162 & $8.03(9.47)$ & 1.04 \\
\hline Yes & 12 & $0.36(0.39)^{*}$ & & 12 & $50.33(21.42)^{*}$ & & 12 & $11.42(6.82)^{*}$ & & 12 & $18.38(16.04)^{*}$ & \\
\hline \multicolumn{13}{|c|}{ Psoriatic arthritis } \\
\hline No & 118 & $0.77(0.24)$ & 1.03 & 121 & $65.61(20.7)$ & 0.44 & 119 & $5.57(6.98)$ & 0.51 & 121 & $6.95(9.12)$ & 0.55 \\
\hline Yes & 56 & $0.48(0.36)^{*}$ & & 57 & $56.61(20.76)^{*}$ & & 57 & $9.26(7.70)^{*}$ & & 57 & $12.42(11.47)^{*}$ & \\
\hline \multicolumn{13}{|c|}{ Localisation of psoriasis } \\
\hline \multicolumn{13}{|c|}{ Visible lesions (on body areas uncovered by clothes) } \\
\hline No & 71 & $0.79(0.24)$ & 0.54 & 72 & $72.1(19.77)$ & 0.6 & 72 & $1.49(3.98)$ & 1.25 & 74 & $2.26(5.24)$ & 1.02 \\
\hline Yes & 113 & $0.63(0.33)^{*}$ & & 116 & $59.75(21.23)^{*}$ & & 114 & $9.3(7.36)^{*}$ & & 118 & $11.34(10.61)^{*}$ & \\
\hline \multicolumn{13}{|c|}{ Facial involvement } \\
\hline No & 144 & $0.74(0.28)$ & 0.55 & 147 & $66.82(20.73)$ & 0.46 & 145 & $4.63(6.48)$ & 0.98 & 150 & $5.65(8.0)$ & 1.04 \\
\hline Yes & 48 & $0.57(0.37)^{*}$ & & 49 & $57.23(21.75)^{*}$ & & 49 & $11.2(7.38)^{*}$ & & 50 & $15.1(12.01)^{*}$ & \\
\hline \multicolumn{13}{|c|}{ Neck and/or décolletage involvement } \\
\hline No & 156 & $0.74(0.28)$ & 0.89 & 160 & $67.75(20.26)$ & 0.9 & 158 & $4.47(6.28)$ & 1.59 & 164 & $5.37(7.63)$ & 1.77 \\
\hline Yes & 36 & $0.48(0.34)^{*}$ & & 36 & $49.65(19.97)^{*}$ & & 36 & $14.28(5.95)^{*}$ & & 36 & $20.01(10.89)^{*}$ & \\
\hline \multicolumn{13}{|c|}{ Psoriasis on hands and/or palms } \\
\hline No & 111 & $0.75(0.26)$ & 0.46 & 114 & $68.41(20.08)$ & 0.46 & 113 & $3.96(6.38)$ & 0.83 & 117 & $4.61(7.24)$ & 0.9 \\
\hline Yes & 81 & $0.61(0.35)^{*}$ & & 82 & $58.88(21.92)^{*}$ & & 81 & $9.53(7.27)^{*}$ & & 83 & $12.8(11.38)^{*}$ & \\
\hline \multicolumn{13}{|c|}{ Psoriasis on hand nails } \\
\hline No & 127 & $0.74(0.28)$ & 0.46 & 128 & $67.06(21.76)$ & 0.36 & 128 & $4.58(6.7)$ & 0.73 & 131 & $6.19(9.52)$ & 0.55 \\
\hline Yes & 65 & $0.60(0.35)^{*}$ & & 68 & $59.47(19.76)^{*}$ & & 66 & $9.61(7.29)^{*}$ & & 69 & $11.47(10.09)^{*}$ & \\
\hline \multicolumn{13}{|c|}{ Medical history } \\
\hline \multicolumn{13}{|c|}{ GP visit(s) in the last month due to psoriasis } \\
\hline No & 145 & $0.77(0.27)$ & 1.05 & 148 & $68.46(20.05)$ & 0.82 & 146 & $4.67(6.37)$ & 0.98 & 151 & $6.52(9.33)$ & 0.63 \\
\hline Yes & 47 & $0.47(0.32)^{*}$ & & 48 & $51.99(20.58)^{*}$ & & 48 & $11.21(7.76)^{*}$ & & 49 & $12.59(10.74)^{*}$ & \\
\hline \multicolumn{13}{|c|}{ Hospitalisation(s) in the last 12 months due to psoriasis } \\
\hline No & 138 & $0.74(0.28)$ & 0.5 & 140 & $68.82(19.52)$ & 0.76 & 138 & $4.76(6.36)$ & 0.77 & 143 & $6.58(9.83)$ & 0.52 \\
\hline Yes & 54 & $0.59(0.36)^{*}$ & & 56 & $53.44(21.91)^{*}$ & & 56 & $10.05(8.08)^{*}$ & & 57 & $11.61(9.64)^{*}$ & \\
\hline \multicolumn{13}{|c|}{ Use of home help (professional or informal) in the last month } \\
\hline No & 165 & $0.75(0.25)$ & 1.45 & 169 & $66.31(20.78)$ & 0.66 & 167 & $5.09(6.66)$ & 1.3 & 173 & $6.49(8.69)$ & 1.22 \\
\hline Yes & 27 & $0.35(0.41)^{*}$ & & 27 & $52.65(21.43)^{*}$ & & 27 & $13.7(6.70)^{*}$ & & 27 & $17.77(12.40)^{*}$ & \\
\hline \multicolumn{13}{|c|}{ Biological therapy } \\
\hline No & 90 & $0.63(0.31)$ & 0.37 & 93 & $57.46(18.35)$ & 0.66 & 93 & $10.8(7.4)$ & 1.48 & 97 & $13.87(10.72)$ & 1.39 \\
\hline Yes & 102 & $0.75(0.31)^{*}$ & & 103 & $70.72(21.96)^{*}$ & & 101 & $2.14(3.92)^{*}$ & & 103 & $2.5(4.91)^{*}$ & \\
\hline
\end{tabular}

* Significant $(p<0.05)$ in Mann-Whitney $U$ test; ** Minimum important difference: 0.09 EQ-5D index score, Shikiar et al. [31]

variance and $30.4 \%$ of EQ VAS variance (adjusted $R^{2}=0.488$ and 0.304, ANOVA $p<0.001$ ). Consequently, mapping functions of the two indices are more accurate than they there were in our bivariate regressions. Three predictors were included in both target variables' model, hospitalisation(s) in the last 12 months, the GP visit(s) in the last month, and presence of palmoplantar involvement.
Furthermore, we noted that global DLQI score did not have an impact on EQ VAS values. However, we found that patients' self-assessed disease severity is implied in the multiple model of EQ VAS with an unstandardized regression coefficient $(\beta)$ of -0.14 . Hence, 1 point fall on the patients' self-assessed VAS eventuates 0.14 point fall in EQ VAS.
333

334

335

336

337

338

339

\begin{tabular}{|l|lll|}
\hline & Journal : Large 10198 & Dispatch : 2-5-2014 & Pages : 9 \\
Article No. : $\mathbf{6 0 0}$ & $\square$ LE & $\square$ TYPESET \\
& MS Code : Supplement14_Paper12 & $\sim_{\text {CP }}$ & $\checkmark$ DISK \\
\hline
\end{tabular}


Table 4 Regression coefficients in the multivariate mapping on EQ-5D and EQ-5D VAS

\begin{tabular}{|c|c|c|c|c|c|c|}
\hline & \multicolumn{3}{|l|}{ EQ-5D score } & \multicolumn{3}{|l|}{ EQ VAS } \\
\hline & $\begin{array}{l}\text { Unstandardized } \\
\text { regression coefficient } \\
(\beta)\end{array}$ & $\begin{array}{l}\text { Standardized } \\
\text { regression } \\
\text { coefficient }\end{array}$ & $p$ & $\begin{array}{l}\text { Unstandardized } \\
\text { regression coefficient } \\
(\beta)\end{array}$ & $\begin{array}{l}\text { Standardized } \\
\text { regression } \\
\text { coefficient }\end{array}$ & $p$ \\
\hline Constant & 1.026 & & $<0.001$ & 110.588 & & $<0.001$ \\
\hline Age & - & - & - & -0.350 & -0.214 & 0.002 \\
\hline Gender (female) & -0.090 & -0.145 & 0.014 & - & - & - \\
\hline BMI & - & - & - & -0.600 & -0.157 & 0.025 \\
\hline Psoriasis duration & -0.004 & -0.169 & 0.006 & - & - & - \\
\hline DLQI & -0.080 & -0.190 & 0.023 & - & - & - \\
\hline $\begin{array}{l}\text { Self-assessed disease severity } \\
\text { VAS }\end{array}$ & - & - & - & -0.14 & -0.218 & 0.004 \\
\hline Chronic plaque psoriasis & -0.089 & -0.151 & 0.029 & - & - & - \\
\hline Palmoplantar psoriasis & -0.347 & -0.269 & $<0.001$ & -12.570 & -0.145 & 0.034 \\
\hline Scalp psoriasis & 0.152 & 0.252 & 0.001 & - & - & - \\
\hline Psoriatic arthritis & -0.134 & -0.212 & 0.002 & - & - & - \\
\hline $\begin{array}{l}\text { GP visit(s) due to psoriasis in the } \\
\text { last month }\end{array}$ & -0.160 & -0.227 & $<0.001$ & -8.112 & -0.167 & 0.022 \\
\hline $\begin{array}{l}\text { Hospitalisation(s) due to } \\
\text { psoriasis in the last } 12 \text { months }\end{array}$ & -0.104 & -0.160 & 0.013 & -12.075 & -0.253 & $<0.001$ \\
\hline $\begin{array}{l}\text { Use of home help (professional } \\
\text { or informal) in the last month }\end{array}$ & -0.139 & -0.160 & 0.021 & - & - & - \\
\hline
\end{tabular}

\section{Discussion}

In this present study, our first purpose was to analyse correlations between quality of life and disease severity measures, and compare their ability in detecting differences between known groups in a sample of 200 moderate to severe psoriasis patients of two Hungarian university clinics.

As a result of the correlation analysis, we found the expected significant correlations between EQ-5D, DLQI, PASI, PGA, and self-assessed disease severity VAS. All the included outcomes correlated only moderately with EQ-5D $\left(r_{\mathrm{s}}=0.41-0.48, p<0.05\right)$. DLQI global score correlated stronger with PASI, PGA, and with self-assessed disease severity, than with EQ-5D.

To date, there are only a few cross-sectional studies in the literature reporting correlation results on outcomes measures in psoriasis. Similarly to our results, Norlin et al. [8] in a sample of 2,450 patients across Sweden found EQ-5D and DLQI moderately correlated $\left(r_{\mathrm{s}}=-0.55\right.$, $p<0.001)$. This is further supported by a survey including 273 patients from Finland where authors observed moderate correlation between EQ-5D and DLQI $(r=-0.52$, $p<0.001)$ [11]. Hjortsberg et al. also pointed out that DLQI score was more highly correlated with patients' selfassessed disease severity than with the EQ-5D $(r=0.71$, $p<0.001)$, likewise in our study $\left(r_{\mathrm{s}}=0.8, p<0.05\right)$.
Two observational studies reported a weak correlation between PASI and EQ-5D $(r=-0.17,-0.25)[8,9]$. In contrast, we noted moderate correlation $(r=-0.43)$ between these two measures. It is, therefore, likely that different clinical protocols of the countries and different patient characteristics of the samples (e.g., psoriasis severity, rate of biological treatment) account for the imparity.

Despite prior evidences that found significant moderate correlations $(r=0.51,0.54)$ between PASI and DLQI, we observed strong correlation $\left(r_{\mathrm{s}}=0.81\right)$ between these two instruments $[8,17]$. We assume that major reasons for the differences are the distinctions amongst the types of treatment (e.g., the proportion of patients on biologicals) and psoriasis severity of the patients included. This assumption is confirmed by the evidence that we demonstrated stronger correlation between DLQI and PASI scores amongst the patients treated with biologicals $\left(r_{\mathrm{s}}=0.76 \mathrm{vs}\right.$ $0.53, p<0.001)$. Furthermore, possible difficulties were described in the comparison of DLQI records related to the patients' different cultural backgrounds. Findings of Nijsten et al. [18] suggest that patients from different countries respond differently to a substantial proportion of DLQI items, although they have the same HRQOL impairment.

A recently conducted systematic review examined the correlation between DLQI and PASI throughout clinical trials of biological agents [19]. Based on 13 randomised

\begin{tabular}{|l|lll|}
\hline & Journal : Large 10198 & Dispatch : 2-5-2014 & Pages : 9 \\
Article No: : $\mathbf{6 0 0}$ & $\square_{\mathrm{LE}}$ & $\square$ \\
MS Code : Supplement14_Paper12 & $\mho_{\mathrm{CP}}^{\text {TYPESET }}$ \\
\hline
\end{tabular}


controlled trials (RCT), the proportion of PASI improvement revealed a strong correlation $(r=0.8)$ with DLQI from the baseline to the 10-16 weeks of treatment, confirming our findings, where more than half of the enrolled patients received biological therapy.

In our study, the highest correlation $\left(r_{\mathrm{s}}=0.92\right.$, $p<0.05)$ was observed between PASI and PGA VAS. Both measures are commonly used in clinical trials. Our finding is consistent with a review based on 30 biological RCTs [20]. According to the results of Robinson et al. [20] the two outcome tools, PGA 0,1 and PASI 75 were correlated very closely $(r=0.9157$ for study weeks $8-16$; $r=0.892$ for weeks $17-24$, and $r=0.9559$ for longer than 24 weeks, $p<0.01$ ).

In the comparison of outcome measures with the knowngroups method, 11 aspects of psoriasis severity were involved, including clinical types, localisations, and health service utilisation variables. A similar method was applied by Revicki et al. [21] validating the psoriasis symptom inventory (PSI), by Dauden et al. [22] validating the PSOLIFE questionnaire, and by Brodszky et al. [23] assessing the Psoriatic Arthritis Quality of Life (PsAQoL) questionnaire and the Health Assessment Questionnaire (HAQ) in psoriatic arthritis. Each of the evaluated tools (see Table 3) was found to be an effective instrument, which was able to discriminate between these groups regarding the severity of psoriasis. Merely a modest effect size was found within the group of hospitalisation(s), similarly to prior results of a study conducted by Brodszky et al. [23] with the same method in psoriatic arthritis, also in Hungary.

The effectiveness of the four assessed tools in tackling QOL varies in different segments. EQ-5D was found remarkably effective from the viewpoint of general HRQOL grouping variables such as the necessity of home help, since the ability for self-care is one of the dimensions of the EQ-5D index. Focusing on strengths of the diseasespecific measures, the discriminating power of DLQI proved the greatest or the second greatest in nine out of the 11 implied categories. In addition, DLQI scores correlated stronger with PASI, patients' self-assessed disease severity and with PGA as well than EQ-5D. Therefore, DLQI is an optimal choice to measure general HRQOL and skin-related symptoms assembled. Not surprisingly, PASI was found especially effective in the distinction of the aspects of visible lesions, localisation of psoriasis, palmoplantar involvement, and biological therapy, because these variables are directly related to disease severity. The presence of visible lesions was analysed with the same method, but with a different instrument (PSO-LIFE) by Daudén et al. [24]. Similarly to our findings, the authors suggest that HRQOL impairment perceived by patients with visible lesions is greater than the effect reported by patients with less visible lesions [24].
Furthermore, we assessed HRQOL in patients with the presence or lack of lesions on certain body regions. The neck and/or décolletage involvement was associated with the greatest EQ-5D reduction, followed by the forearm, and facial lesions. Also, the neck and/or décolletage involvement proved the highest effect size in DLQI scores, followed by the forearm, and the leg and/or shin lesions. Unexpectedly, the effect sizes of the facial psoriasis, which is likely the most bothersome localisation due to stigmatisation and cosmetic issues, were overtaken by the neck and/or décolletage measured by any examined outcome. We assume that this is due to the fact that in our sample the majority of the patients with neck and/or décolletage involvement $(n=36)$ had lesions on two or more body sites, covering a higher proportion of their entire body surface.

Our second aim was to investigate new possible predictors of EQ-5D score and EQ VAS, and seek for a mapping algorithm for these variables. Bivariate analysis on EQ-5D was previously published in two studies. A simple linear regression developed by Currie and Conway [10] amongst 94 patients could account for $27 \%$ of EQ-5D variance: $\mathrm{EQ}-5 \mathrm{D}=0.956-0.02548 * \mathrm{DLQI}$. The model of Norlin et al. [8] was able to explain $28 \%$ of the EQ-5D variance $(\mathrm{EQ}-5 \mathrm{D}=0.8777-0.0196 * \mathrm{DLQI})$. Our model is in line with these two bivariate algorithms, the constant term is about 0.8 and one point increase in DLQI is expected to result in a reduction of 0.02 point in EQ-5D.

A study from Germany including 1,511 patients performed by Blome et al. [9] could predict $24.2 \%$ of the variability of EQ VAS with the following mapping algorithm: $\mathrm{EQ} \quad \mathrm{VAS}=77.367-1.493 * \mathrm{DLQI} \quad(p<0.001)$. Furthermore, these results were cross-validated by a database of 2,009 patients.

To develop our multivariate function, we explored ten variables as possible predictors of EQ-5D: DLQI, gender, psoriasis duration, palmoplantar involvement, psoriatic arthritis, chronic plaque psoriasis, scalp psoriasis, necessity of home help in the last month, GP visit(s) due to psoriasis in the last month, and hospitalisation(s) due to psoriasis in the last 12 months. The clinical type of palmoplantar involvement had the greatest negative standardized regression coefficient. This finding seems to be consistent with earlier researches, which described that patients with palmoplantar involvement have reported significantly greater physical disability, discomfort, and work or leisure impairment than those without palmoplantar involvement $[25,26]$. In contrast, scalp psoriasis was the only variable with positive unstandardized regression coefficient $(\beta)$ involved in the model. This might be conceivably due to the high proportion of the less severe cases amongst the patients of our sample with scalp involvement $(n=69)$, and, therefore, this finding cannot be generalised. 
In the multivariate approach of Norlin et al. [8], in addition to DLQI (global score or single items) gender and age were found to be predictors of EQ-5D. Their model could explain $32 \%$ of the variance of EQ-5D.

Blome et al. [9] implemented a stepwise linear regression on EQ-5D as well as on EQ-5D VAS with powers of explanation of 27.9 and $31.3 \%$. Age, presence of active arthritis and concomitant diseases predicted both target variables. Gender, psoriasis duration, and nail involvement were also described as predictors of EQ-5D. Compared to our model, gender, psoriatic arthritis, and disease duration are common predictors. The regression coefficients of DLQI are higher in both the bivariate and the multivariate function of Blome et al., than in ours [9].

It seems that gender is the only variable that was found as a predictor in the two referred multivariate mapping functions and also in our model $[8,9]$. A literature review on quality of life in psoriasis patients points out that there is no association between gender and HRQOL in psoriasis [2]. However, a few authors have described higher HRQOL impairment in female patients, possibly caused by stigmatisation and additional mental disorders [17, 27]. Lesuis et al. [28] also indicated that men more often had high PASI scores and women more often had high DLQI scores. In our study we could not justify significant difference neither in DLQI nor in PASI index, nonetheless, median EQ-5D in female patients was significantly worse than in males $(0.67$ vs $0.8, p<0.001)$.

Mapping EQ VAS, we observed that self-assessed disease severity VAS overwhelmed DLQI as a possible predictor, and hence, confirmed the importance of selfassessed disease severity as an outcome measure, as earlier also highlighted by Hjortsberg et al. [11].

To summarise, the three cited bivariate models can predict a greater proportion of the variance of EQ-5D or EQ VAS than our mapping functions. However, our multiple linear regression algorithm can predict $48.8 \%$ of EQ$5 \mathrm{D}$ scores, which is more accurate than in any previously published models.

Finally, a number of important limitations need to be considered. To our knowledge, HRQOL median values of our sample are reflecting better health states than in other previous cross-sectional surveys. This might be the result of the biological treatment received by about half of our patients and also due to the treatment institutions, which were two university clinics considered to offer higher quality of care. Additionally, several limitations of mapping should be noted. Sample size was relatively small, only the ordinary least squares method was applied and no cross-validation was conducted. A recently published study suggests that the ordinary least squares method systematically underestimates mapping from disease-specific measures, like DLQI to generic measures such as EQ-5D [29].
Consequently, the developed mapping algorithm is probably not transferable to all Hungarian psoriasis patients, merely to subgroups of patients.

A broader survey including more variables not investigated in this study (e.g., time on biological treatment, comorbidities and concomitant medications, mental health, body image, coping mechanisms) is needed to reduce the uncertainties around the model and to determine the still unexplained $51.2 \%$ of EQ-5D. A detailed analysis in terms of the individual five dimensions of EQ-5D and of each DLQI questions or items might as well improve the predictive power of mapping [30].

This current study confirms previous findings about correlations between EQ-5D, EQ VAS, DLQI, and PASI. We provided the first evidence that visible psoriatic lesions have a significant impact on HRQOL measured not only with DLQI, but also with EQ-5D, compared to non-visible skin lesions. We revealed new possible predictors of HRQOL, such as clinical types and localisation of psoriasis, and the necessity of home help in patients with moderate to severe psoriasis. In clinical trials, when direct utility outcomes are not available, our mapping functions can contribute to the valuation of utilities. Notwithstanding the limitations listed above, predictors tested in a multivariate approach explained a higher proportion of variance of EQ-5D in psoriasis than any other models before.

Acknowledgments This study was supported by The Hungarian Scientific Research Fund (OTKA K108421, TÁMOP-4.2.2.A-11/1/ KONV-2012-0023, TÁMOP-4.2.2.A-11/1/KONV-2012-0031).

\section{References}

1. Parisi, R., Symmons, D.P., Griffiths, C.E., Ashcroft, D.M.: Global epidemiology of psoriasis: a systematic review of incidence and prevalence. J. Invest. Dermatol. 133(2), 377-385 (2013). doi:10. 1038/jid.2012.339

2. de Korte, J., Sprangers, M.A., Mombers, F.M., Bos, J.D.: Quality of life in patients with psoriasis: a systematic literature review. J. Investig. Dermatol. Symp. Proc. 9(2), 140-147 (2004). doi:10. 1046/j.1087-0024.2003.09110.x

3. Ahn, C.S., Gustafson, C.J., Sandoval, L.F., Davis, S.A., Feldman, S.R.: Cost effectiveness of biologic therapies for plaque psoriasis. Am. J. Clin. Dermatol. 14(4), 315-326 (2013). doi:10.1007/ s40257-013-0030-z

4. Brodszky, V.: Systematic Review and Analysis of Evidences on Clinical Efficacy and Cost-Effectiveness of Biological Drugs for the Treatment of Prosiasis. Corvinus University of Budapest, Department of Health Economics, Budapest, Hungary (2013)

5. Bronsard, V., Paul, C., Prey, S., Puzenat, E., Gourraud, P.A., Aractingi, S., Aubin, F., Bagot, M., Cribier, B., Joly, P., Jullien, D., Le Maitre, M., Richard-Lallemand, M.A., Ortonne, J.P.: What are the best outcome measures for assessing quality of life in plaque type psoriasis? A systematic review of the literature. J. Eur. Acad. Dermatol. Venereol. 24(Suppl 2), 17-22 (2010). doi:10.1111/j.1468-3083.2009.03563.x

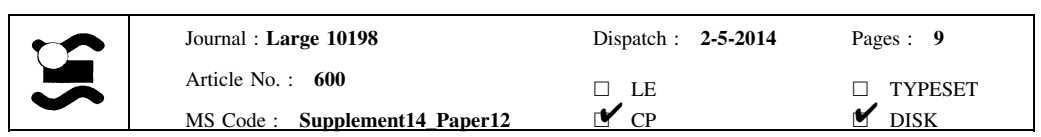


6. Lewis, V., Finlay, A.Y.: 10 years experience of the Dermatology Life Quality Index (DLQI). J. Investig. Dermatol. Symp. Proc. 9(2), 169-180 (2004). doi:10.1111/j.1087-0024.2004.09113.x

7. Dakin, H.: Review of studies mapping from quality of life or clinical measures to EQ-5D: an online database. Health Qual. Life Outcomes 11, 151 (2013). doi:10.1186/1477-7525-11-151

8. Norlin, J.M., Steen Carlsson, K., Persson, U., Schmitt-Egenolf, M.: Analysis of three outcome measures in moderate to severe psoriasis: a registry-based study of 2450 patients. Br. J. Dermatol. 166(4), 797-802 (2012). doi:10.1111/j.1365-2133.2011.10778.x

9. Blome, C., Beikert, F.C., Rustenbach, S.J., Augustin, M.: Mapping DLQI on EQ-5D in psoriasis: transformation of skin-specific health-related quality of life into utilities. Arch. Dermatol. Res. 305(3), 197-204 (2013). doi:10.1007/s00403-012-1309-2

10. Currie, C.J., Conway, P.: Evaluation of the association between EQ5D utility and dermatology life quality index (DLQI) score in patients with psoriasis. Value Health 10(6), A470 (2007)

11. Hjortsberg, C., Bergman, A., Bjarnason, A., Heikkila, H., Hjelmgren, J., Svensson, A., Tennvall, G.R.: Are treatment satisfaction, quality of life, and self-assessed disease severity relevant parameters for patient registries? Experiences from Finnish and Swedish patients with psoriasis. Acta Derm. Venereol. 91(4), 409-414 (2011). doi:10.2340/00015555-1094

12. Dolan, P.: Modeling valuations for EuroQol health states. Med. Care 35(11), 1095-1108 (1997)

13. Finlay, A.Y., Khan, G.K.: Dermatology Life Quality Index (DLQI) - a simple practical measure for routine clinical use. Clin. Exp. Dermatol. 19(3), 210-216 (1994)

14. Basra, M.K., Fenech, R., Gatt, R.M., Salek, M.S., Finlay, A.Y.: The Dermatology Life Quality Index 1994-2007: a comprehensive review of validation data and clinical results. Br. J. Dermatol. 159(5), 997-1035 (2008). doi:10.1111/j.1365-2133.2008. 08832.x

15. Cohen, J.: A power primer. Psychol. Bull. 112, 155-159 (1992)

16. Balogh, O., Brodszky, V., Gulácsi, L., Herédi, E., Herszényi, K., Jókai, H., Kárpáti, S., Baji, P., Remenyik, É., Szegedi, A., Holló, P.: Cost-of-illness in patients with moderate to severe psoriasis: a cross-sectional survey in Hungarian dermatological centres. Eur. J. Health Econ. (2014). doi:10.1007/s10198-014-0599-z

17. Mabuchi, T., Yamaoka, H., Kojima, T., Ikoma, N., Akasaka, E., Ozawa, A.: Psoriasis affects patient's quality of life more seriously in female than in male in Japan. Tokai J. Exp. Clin. Med. 37(3), 84-88 (2012)

18. Nijsten, T., Meads, D.M., de Korte, J., Sampogna, F., Gelfand, J.M., Ongenae, K., Evers, A.W., Augustin, M.: Cross-cultural inequivalence of dermatology-specific health-related quality of life instruments in psoriasis patients. J. Invest. Dermatol. 127(10), 2315-2322 (2007). doi:10.1038/sj.jid.5700875

19. Mattei, P.L., Corey, K.C., Kimball, A.B.: Psoriasis Area Severity Index (PASI) and the Dermatology Life Quality Index (DLQI): the correlation between disease severity and psychological burden in patients treated with biological therapies. J. Eur. Acad. Dermatol. Venereol. (2013). doi:10.1111/jdv.12106

20. Robinson, A., Kardos, M., Kimball, A.B.: Physician Global Assessment (PGA) and Psoriasis Area and Severity Index (PASI): why do both? A systematic analysis of randomized controlled trials of biologic agents for moderate to severe plaque psoriasis. J. Am. Acad. Dermatol. 66(3), 369-375 (2012). doi:10.1016/j. jaad.2011.01.022

21. Revicki, D.A., Jin, Y., Wilson, H.D., Chau, D., Viswanathan, H.N.: Reliability and validity of the psoriasis symptom inventory in patients with moderate-to-severe psoriasis. J. Dermatolog. Treat. 25(1), 8-14 (2014). doi:10.3109/09546634.2013.769042

22. Dauden, E., Herrera, E., Puig, L., Sanchez-Carazo, J.L., Toribio, J., Caloto, M.T., Nocea, G., Roset, M., Lara, N.: Validation of a new tool to assess health-related quality of life in psoriasis: the PSO-LIFE questionnaire. Health Qual. Life Outcomes 10, 56 (2012). doi:10.1186/1477-7525-10-56

23. Brodszky, V., Pentek, M., Balint, P.V., Geher, P., Hajdu, O., Hodinka, L., Horvath, G., Koo, E., Polgar, A., Sesztak, M., Szanto, S., Ujfalussy, I., Gulacsi, L.: Comparison of the Psoriatic Arthritis Quality of Life (PsAQoL) questionnaire, the functional status (HAQ) and utility (EQ-5D) measures in psoriatic arthritis: results from a cross-sectional survey. Scand. J. Rheumatol. 39(4), 303-309 (2010). doi:10.3109/03009740903468982

24. Dauden, E., Herrera, E., Puig, L., Sanchez-Carazo, J.L., Toribio, J., Perulero, N.: Impact of active and stable psoriasis on healthrelated quality of life: the PSO-LIFE study. Actas Dermosifiliogr. 104(8), 685-693 (2013). doi:10.1016/j.adengl.2013.02.008

25. Pettey, A.A., Balkrishnan, R., Rapp, S.R., Fleischer, A.B., Feldman, S.R.: Patients with palmoplantar psoriasis have more physical disability and discomfort than patients with other forms of psoriasis: implications for clinical practice. J. Am. Acad. Dermatol. 49(2), 271-275 (2003)

26. Sampogna, F., Tabolli, S., Abeni, D.: Living with psoriasis: prevalence of shame, anger, worry, and problems in daily activities and social life. Acta Derm. Venereol. 92(3), 299-303 (2012). doi: $10.2340 / 00015555-1273$

27. Sampogna, F., Chren, M.M., Melchi, C.F., Pasquini, P., Tabolli, S., Abeni, D.: Age, gender, quality of life and psychological distress in patients hospitalized with psoriasis. Br. J. Dermatol. 154(2), 325-331 (2006). doi:10.1111/j.1365-2133.2005.06909.x

28. Lesuis, N., Befrits, R., Nyberg, F., van Vollenhoven, R.F.: Gender and the treatment of immune-mediated chronic inflammatory diseases: rheumatoid arthritis, inflammatory bowel disease and psoriasis: an observational study. BMC Med. 10, 82 (2012). doi:10.1186/1741-7015-10-82

29. Lu, G., Brazier, J.E., Ades, A.E.: Mapping from disease-specific to generic health-related quality-of-life scales: a common factor model. Value Health 16(1), 177-184 (2013). doi:10.1016/j.jval. 2012.07.003

30. Brazier, J.E., Yang, Y., Tsuchiya, A., Rowen, D.L.: A review of studies mapping (or cross walking) non-preference based measures of health to generic preference-based measures. Eur. J. Health Econ. 11(2), 215-225 (2010). doi:10.1007/s10198-0090168-Z

31. Shikiar, R., Willian, M.K., Okun, M.M., Thompson, C.S., Revicki, D.A.: The validity and responsiveness of three quality of life measures in the assessment of psoriasis patients: results of a phase II study. Health Qual. Life Outcomes 4, 71 (2006). doi:10. $1186 / 1477-7525-4-71$

\begin{tabular}{|l|lll|}
\hline Journal : Large $\mathbf{1 0 1 9 8}$ & Dispatch : 2-5-2014 & Pages : 9 \\
Article No. : $\mathbf{6 0 0}$ & $\square$ LE & $\square$ TYPESET \\
& MS Code : Supplement14_Paper12 & $\sim_{\text {CP }}$ & $\checkmark$ DISK \\
\hline
\end{tabular}

